\title{
Analytical and Bioanalytical Chemistry
}

Supporting information

\section{AN UNTARGETED METABOLOMIC STRATEGY BASED ON LIQUID CHROMATOGRAPHY-MASS SPECTROMETRY TO STUDY HIGH}

\section{GLUCOSE-INDUCED CHANGES IN CULTURED HUMAN PROXIMAL}

\section{TUBULAR CELLS}

Samuel Bernardo-Bermejo ${ }^{1}$, Elena Sánchez-López ${ }^{1}$, María Castro-Puyana ${ }^{1,2}$, Selma Benito $^{3}$, Francisco Javier Lucio-Cazaña ${ }^{3}$, María Luisa Marina ${ }^{1,2 *}$

${ }^{1}$ Departamento de Química Analítica, Química Física e Ingeniería Química, Universidad de Alcalá, Ctra. Madrid-Barcelona Km. 33.600, 28871 Alcalá de Henares (Madrid), Spain.

${ }^{2}$ Instituto de Investigación Química Andrés M. del Río (IQAR), Universidad de Alcalá, Ctra. Madrid-Barcelona Km. 33.600, 28871 Alcalá de Henares (Madrid), Spain.

${ }^{3}$ Departamento de Biología de Sistemas, Universidad de Alcalá, Ctra. Madrid-Barcelona Km. 33.600, 28871 Alcalá de Henares (Madrid), Spain.

*Correspondence: Departamento de Química Analítica, Química Física e Ingeniería Química, Universidad de Alcalá, Ctra. Madrid-Barcelona, Km. 33.600, 28871 Alcalá de Henares, Madrid, España.

E-mail: mluisa.marina@uah.es

Fax: $+34-918854971$

Tel: +34-91 8854935 


\section{Experimental conditions}

\section{Initial procedure for sample preparation of the intracellular fluid}

To begin with the extraction optimization of intracellular fluid, cell pellets from section 2.2 were extracted with $400 \mu \mathrm{L}$ of $75 \%(v / v) \mathrm{MeOH}$ in water, vortexed for $30 \mathrm{~s}$ and left still for $5 \mathrm{~min}$ in an ultrasonic bath followed by a centrifugation step (14000 $\mathrm{g}$ for 5 min at $4{ }^{\circ} \mathrm{C}$ ). The supernatant was separated into two equal parts of $200 \mu \mathrm{L}$ each and were evaporated for $3.5 \mathrm{~h}$ till dryness. $100 \mu \mathrm{L}$ of $80 \%(\mathrm{v} / \mathrm{v})$ acetonitrile in water were added to the dried samples to be analyzed by HILIC and $100 \mu \mathrm{L}$ of water were added to the dried samples to be analyzed by RPLC, they were vortexed for $30 \mathrm{~s}$, centrifuged at $14000 \mathrm{~g}$ for $5 \mathrm{~min}$ at $4{ }^{\circ} \mathrm{C}$ and supernatants were placed in glass inserts for further analyses.

\section{Initial conditions for intracellular fluid LC-MS analysis}

All initial LC-MS conditions are the same as the ones included in section 2.4, except for the fragmentator which was set at $100 \mathrm{~V}$ both in HILIC and RPLC and that analyses were performed in positive ESI mode for both chromatographic modes. 


\section{Figures}

Figure S1. PCA comparing different data normalization methods: A) dataset normalized to protein content, and B) dataset normalized to cell number.

Figure S2. PCA without QCs for the four analytical sequences. A) intracellular fluid (HILIC), B) intracellular fluid (RPLC), C) extracellular fluid (HILIC), and D) extracellular fluid (RPLC).

Figure S3. Permutation tests of all PLS-DA models.

Figure S4. Box-plots of the five metabolites of the intracellular fluid unequivocally and tentatively identified.

Figure S5. Box-plots of the five metabolites of the extracellular fluid unequivocally and tentatively identified.

Figure S6. Box-plots of the five metabolites of the both fluids unequivocally and tentatively identified. 


\section{Figure S1.}

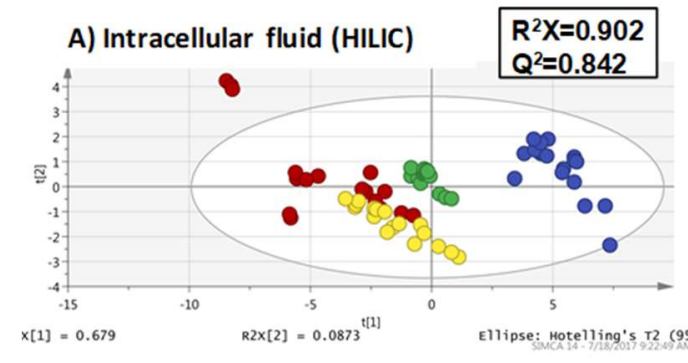

Normalized to protein content

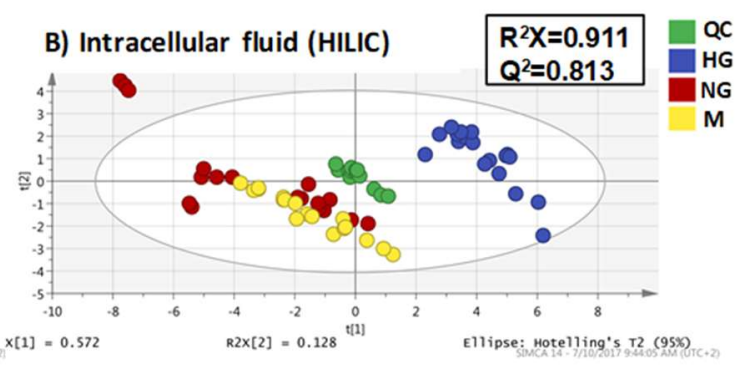

Normalized to the cell number 


\section{Figure S2.}
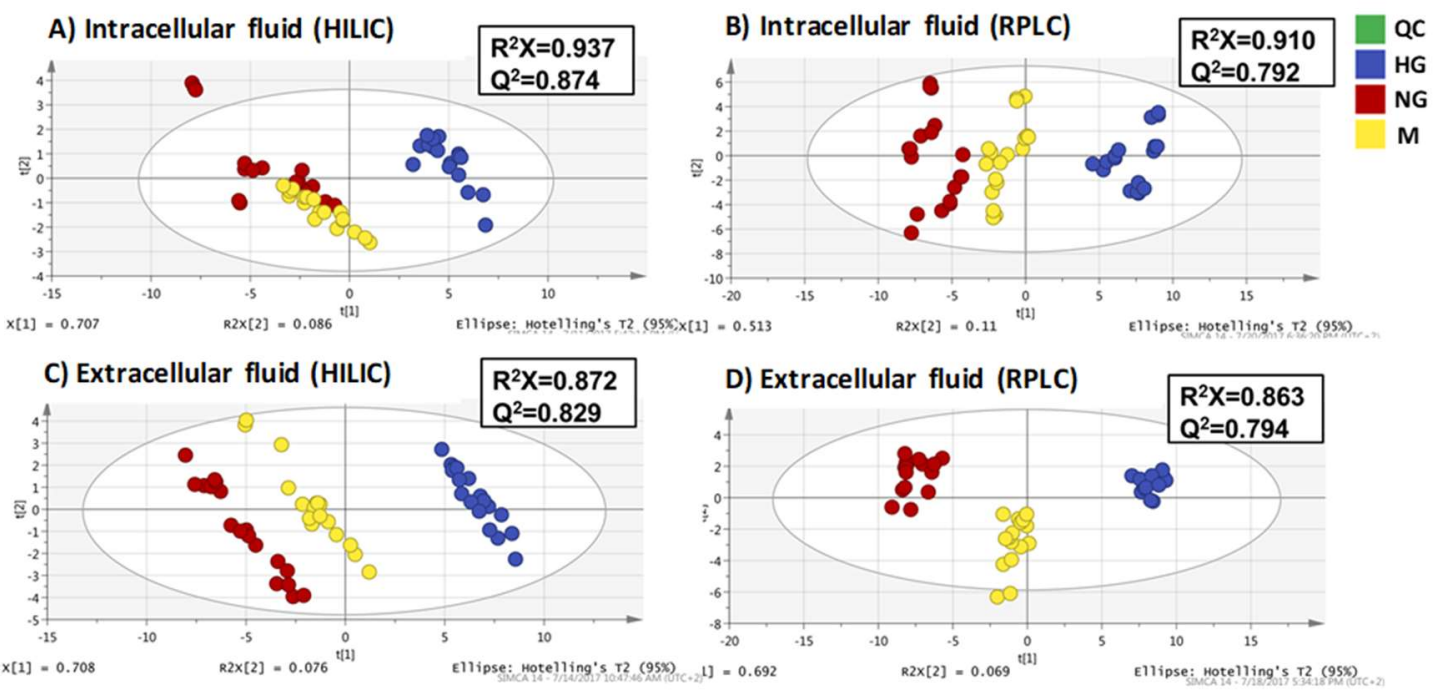


\section{Figure S3.}

\section{PLS-DA (HG vs NG)}

A) Intracellular fluid (HILIC)

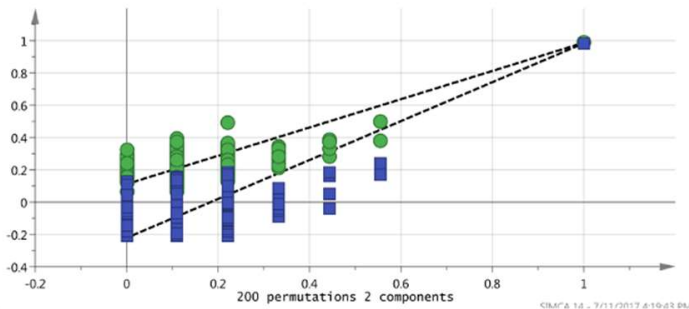

C) Extracellular fluid (HILIC)

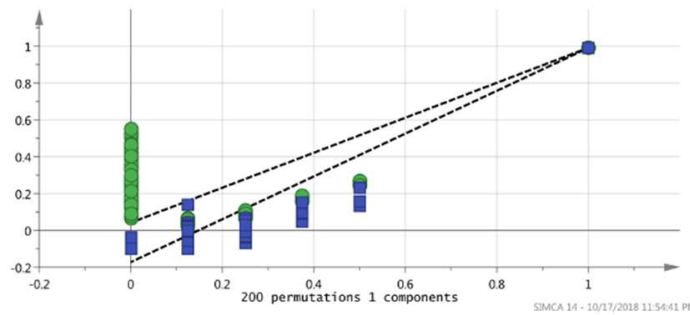

B) Intracellular fluid (RPLC)

R2

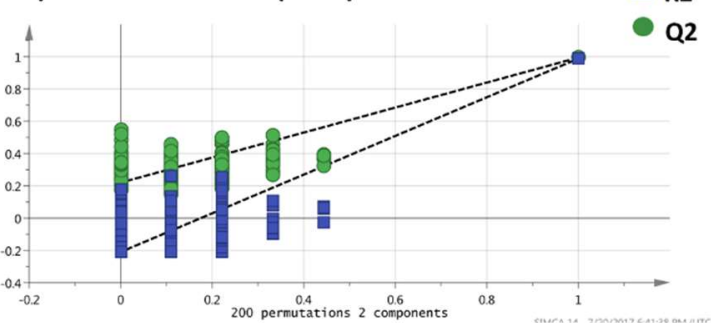

D) Extracellular fluid (RPLC)

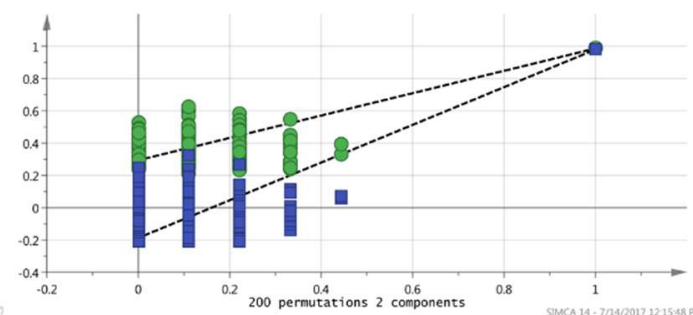

PLS-DA (NG vs M)

E) Intracellular fluid (HILIC)

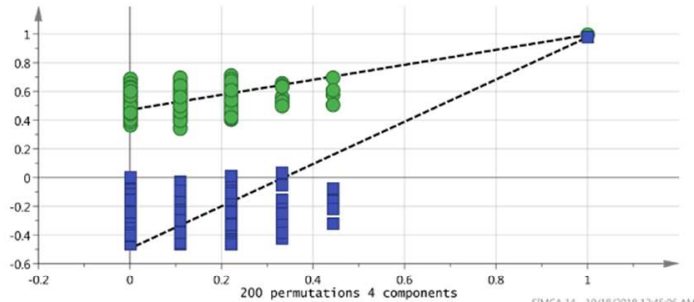

G) Extracellular fluid (HILIC)

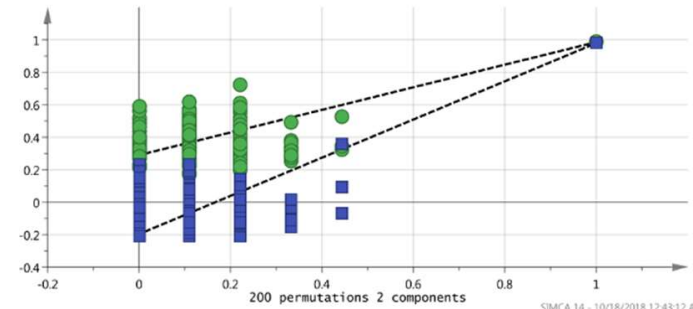

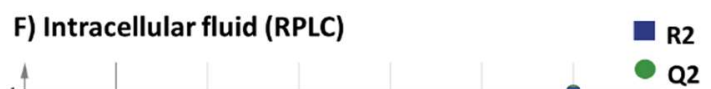

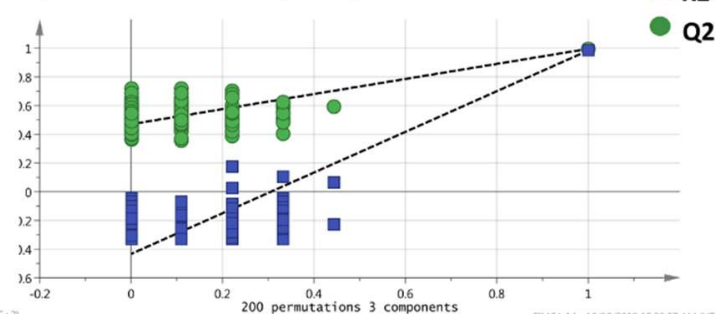

H) Extracellular fluid (RPLC)

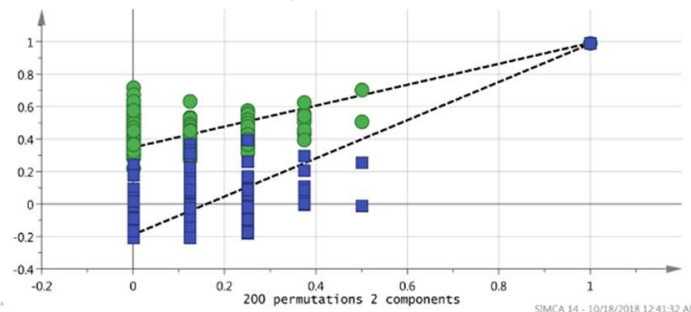


Figure S4.
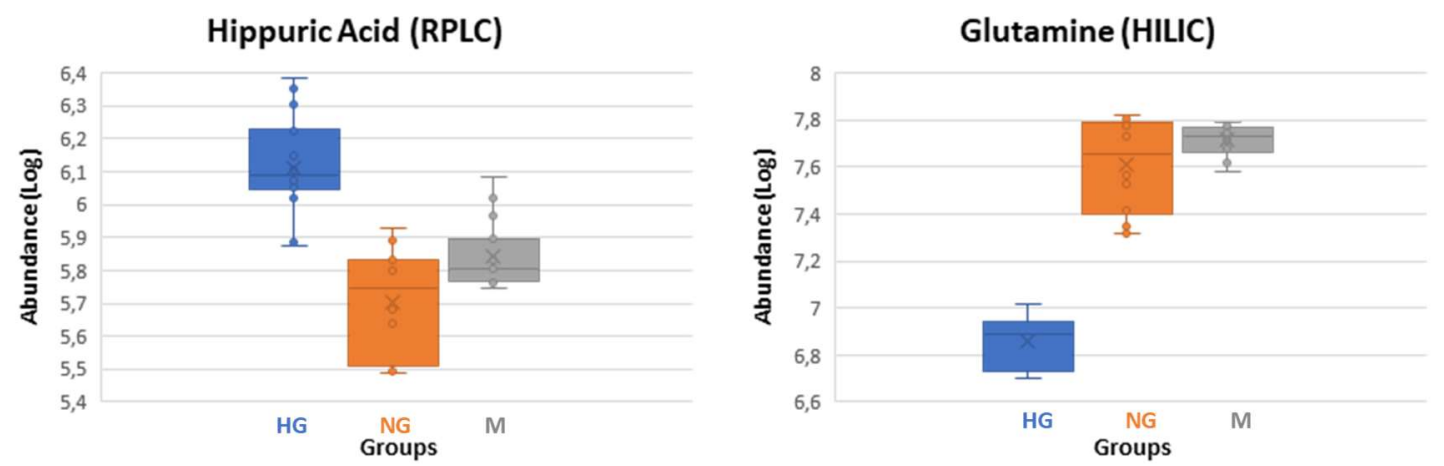

Sorbitol (HILIC)

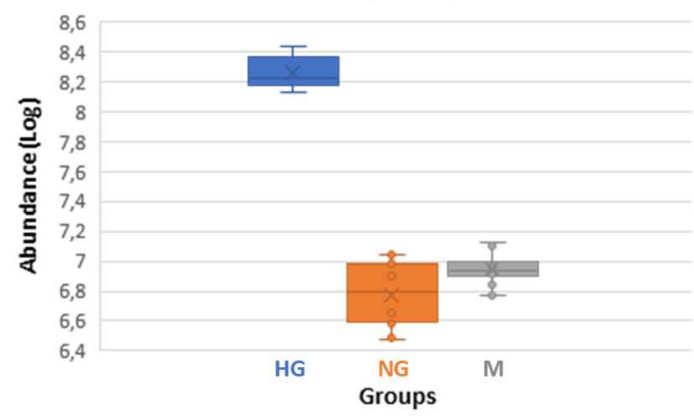

$\mathrm{N}$-steaoryl valine (RPLC)

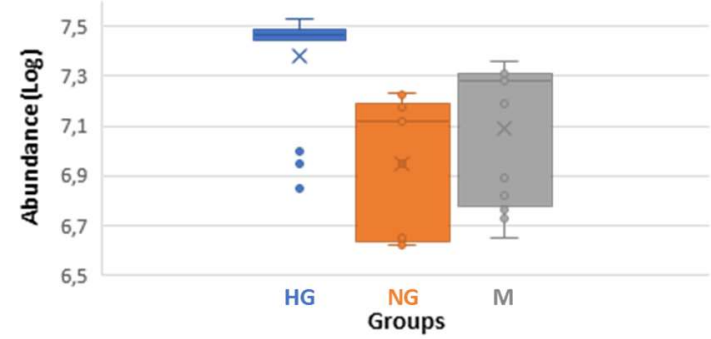

L-4-Hydroxy-3-methoxy-a-

methylphenylalanine (RPLC)

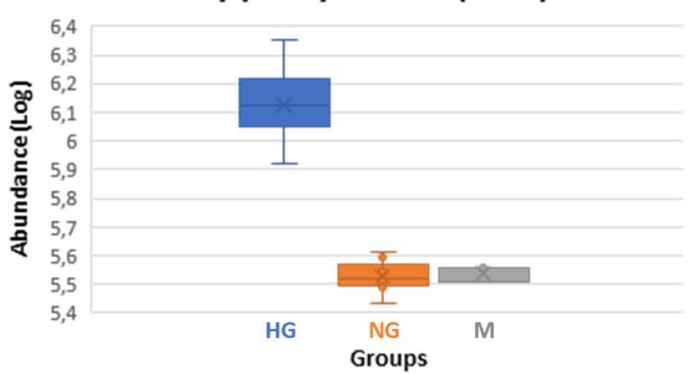




\section{Figure S5.}
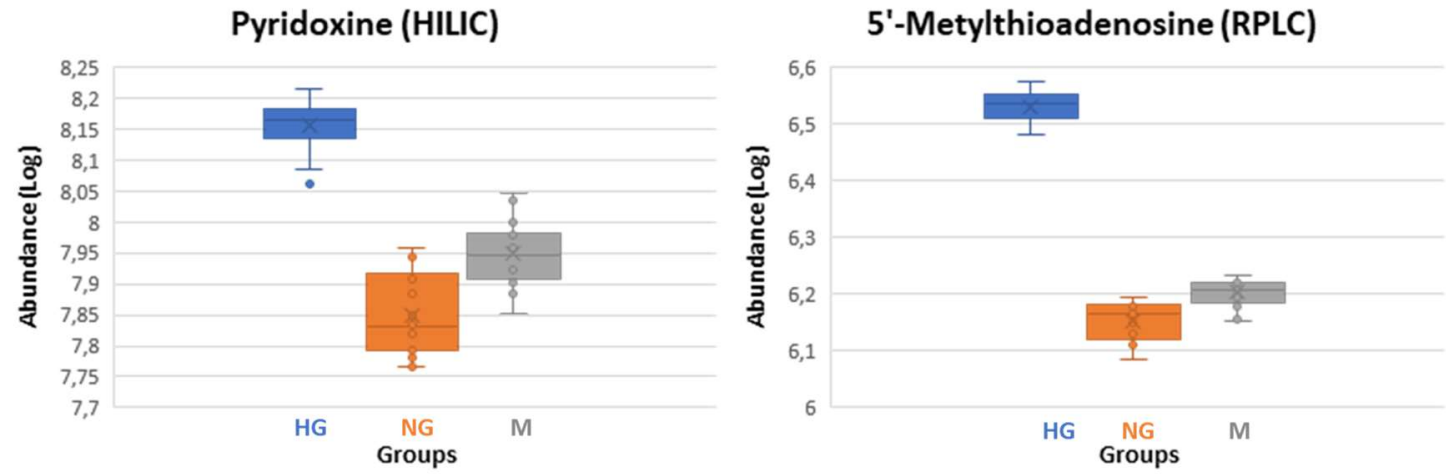

$\mathrm{N}$-(1-Deoxy-1-fructosyl)leucine or N-(1-

Deoxy-1-fructosyl)isoleucine (RPLC)

\section{$\mathrm{N}$-(1-Deoxy-1-fructosyl)phenylalanine}

(RPLC)
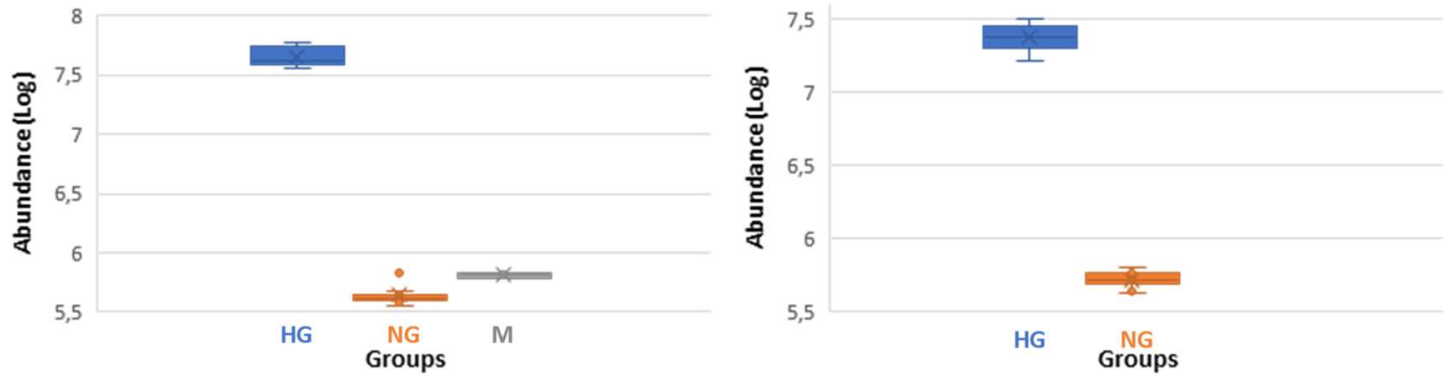

Taurodeoxycholic acid or taurochenodeoxycholic acid (HILIC)

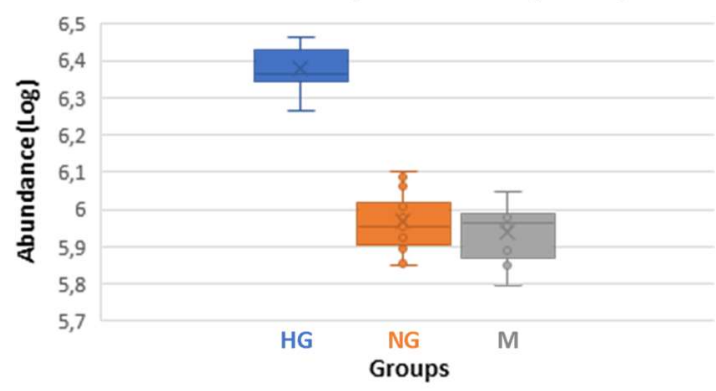




\section{Figure S6.}

Phenylacetylglycine (HILIC; extracellular fluid)

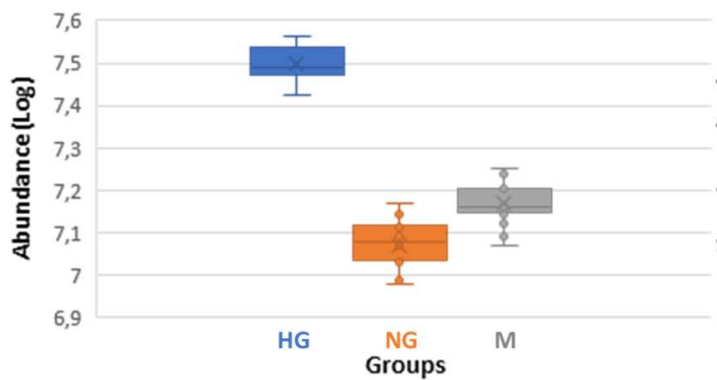

Phenylacetylglycine (RPLC; extracellular fluid)

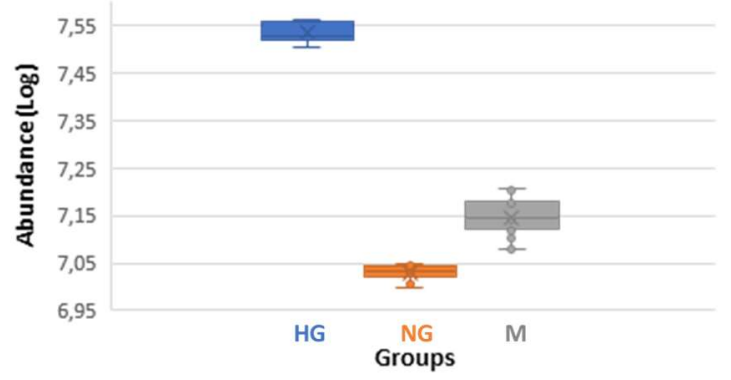

Pyroglutamic acid (HILIC; extracellular fluid)

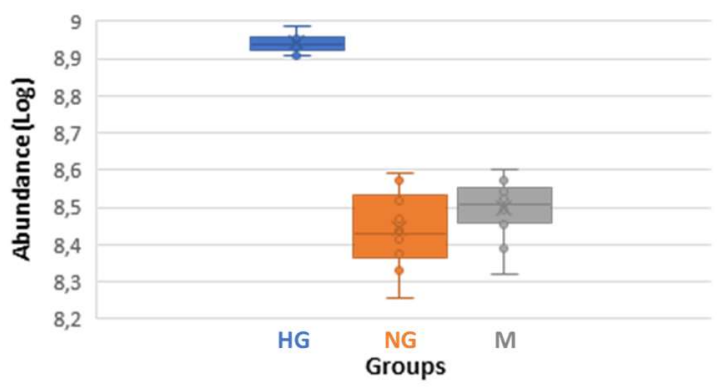

Phenylacetylglycine (HILIC; intracellular fluid)

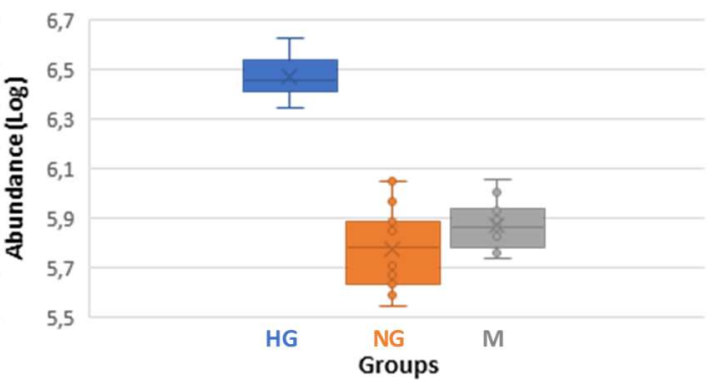

Pyroglutamic (HILIC; intracelular fluid)

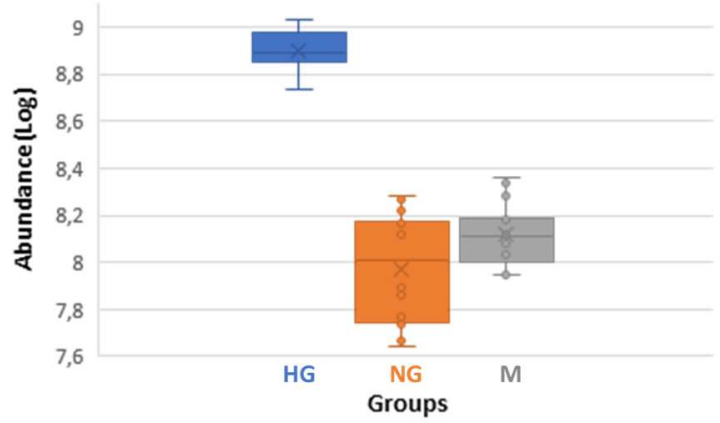


Table S1. Unknown molecular features which were statistically significant for any of the four analytical sequences.

\begin{tabular}{|c|c|c|c|c|c|c|}
\hline \multirow{2}{*}{$\#$} & \multirow{2}{*}{ RT (min) } & \multirow{2}{*}{$\begin{array}{c}\text { Monoisotopic } \\
\text { mass (Da) }\end{array}$} & \multirow{2}{*}{ Main fragments (MS/MS) } & \multicolumn{2}{|c|}{ VIP } & \multirow{2}{*}{ Trend* } \\
\hline & & & & HG vs NG & HG vs NG & \\
\hline \multicolumn{7}{|c|}{ Intracellular fluid (HILIC) } \\
\hline 1 & 2.66 & 148.0734 & - & 1.25 & 0.93 & $\uparrow$ \\
\hline 2 & 4.81 & 758.4309 & - & 1.25 & 0.78 & $\uparrow$ \\
\hline 3 & 7.26 & 272.1126 & - & 1.53 & 0.74 & $\uparrow$ \\
\hline 4 & 7.62 & 581.3795 & - & 1.36 & 0.68 & $\uparrow$ \\
\hline 5 & 7.62 & 296.0426 & $259.0822,223.0121,189.9783,149.0022$ & 1.33 & 0.23 & $\uparrow$ \\
\hline 6 & 7.62 & 807.5493 & - & 1.39 & 0.63 & $\uparrow$ \\
\hline 7 & 7.63 & 447.0679 & - & 1.29 & 0.78 & $\uparrow$ \\
\hline 8 & 7.63 & 598.0909 & - & 1.26 & 0.83 & $\uparrow$ \\
\hline 9 & 7.63 & 425.1665 & - & 1.37 & 0.55 & $\uparrow$ \\
\hline 10 & 7.64 & 280.0683 & $128.0363,146.9625$ & 1.40 & 0.78 & $\uparrow$ \\
\hline 11 & 7.65 & 309.1076 & - & 2.01 & 0.45 & $\uparrow$ \\
\hline 12 & 7.65 & 471.1899 & $198.0131,451.0046,306.9910,362.9814$ & 1.42 & 0.67 & $\uparrow$ \\
\hline 13 & 7.81 & 187.0021 & - & 1.21 & 0.94 & $\uparrow$ \\
\hline 14 & 11.64 & 374.0922 & $229.1363,285.1041,248.9958,137.0751$ & 1.32 & 0.32 & $\uparrow$ \\
\hline 15 & 11.64 & 308.1201 & $229.1359,137.0760,117.0373,266.9887$ & 1.46 & 0.81 & $\uparrow$ \\
\hline 16 & 14,31 & 314.1209 & $145.0616,127.0518,109.0403$ & 1.27 & 0.77 & $\downarrow$ \\
\hline 17 & 14.31 & 488.2177 & $305.1180,145.0639,227.0870,341.1378$ & 1.10 & 0.97 & $\downarrow$ \\
\hline \multicolumn{7}{|c|}{ Intracellular fluid (RPLC) } \\
\hline 18 & 0.82 & 302.0502 & 174.0116 & 1.52 & 0.79 & $\uparrow$ \\
\hline 19 & 1.14 & 262.0894 & $122.0602,160.0057,214.1103,179.0990$ & 1.30 & 0.74 & $\uparrow$ \\
\hline 20 & 1.29 & 471.2808 & - & 1.28 & 0.21 & $\uparrow$ \\
\hline 21 & 1.44 & 514.3223 & $258.1521,138.0466,498.3221$ & 1.36 & 0.48 & $\uparrow$ \\
\hline 22 & 1.79 & 676.3577 & $281.1392,504.3969,171.0762,129.1048$ & 1.16 & 0.60 & $\uparrow$ \\
\hline 23 & 2.02 & 693.3844 & - & 1.23 & 0.28 & $\uparrow$ \\
\hline 24 & 4.18 & 348.1436 & $188.0679,146.0613,159.0922,117.0637$ & 1.26 & 0.50 & $\uparrow$ \\
\hline 25 & 4.23 & 722.2880 & - & 1.14 & 0.71 & $\uparrow$ \\
\hline 26 & 4.32 & 287.1844 & $143.1195,157.8862,129.0979$ & 1.43 & 0.33 & $\uparrow$ \\
\hline 27 & 5.21 & 345.1902 & $187.1087,127.0874,142.0884,169.0973$ & 1.18 & 0.46 & $\uparrow$ \\
\hline 28 & 5.33 & 606.2323 & $230.0784,345.0946,328.0754,201.1194$ & 1.14 & 0.31 & $\uparrow$ \\
\hline 29 & 5.41 & 391.2107 & 279.1338 & 1.29 & 0.88 & $\uparrow$ \\
\hline
\end{tabular}




\begin{tabular}{|c|c|c|c|c|c|c|}
\hline 30 & 5.69 & 321.1690 & 187.1046 & 1.24 & 0.54 & $\uparrow$ \\
\hline 31 & 5.78 & 851.4043 & - & 1.11 & 0.73 & $\uparrow$ \\
\hline 32 & 6.30 & 466.1909 & $129.0994,102.0574$ & 1.58 & 0.44 & $\uparrow$ \\
\hline 33 & 6.49 & 480.2062 & $322.1741,147.1110,130.0898$ & 1.59 & 0.75 & $\uparrow$ \\
\hline 34 & 6.49 & 321.1687 & $253.1559,270.1896,201.8895,114.0312$ & 1.57 & 0.79 & $\uparrow \uparrow$ \\
\hline 35 & 6.49 & 159.0349 & $114.0386,102.0098,118.09864$ & 1.55 & 0.67 & $\uparrow$ \\
\hline 36 & 6.68 & 341.2309 & $229.1560,183.1514$ & 1.20 & 0.85 & $\uparrow$ \\
\hline 37 & 7.23 & 480.2059 & $463.2520,295.0297,129.1016$ & 1.82 & 0.73 & $\uparrow$ \\
\hline 38 & 7.70 & 572.3173 & - & 1.17 & 0.14 & $\uparrow$ \\
\hline 39 & 7.91 & 439.1416 & - & 1.22 & 0.75 & $\uparrow$ \\
\hline 40 & 7.95 & 537.3520 & $120.0790,508.6301,484.6276,521.2505$ & 1.12 & 0.53 & $\uparrow$ \\
\hline 41 & 7.98 & 660.2289 & $614.0418,561.7185,581.3516$ & 1.20 & 0.30 & $\uparrow$ \\
\hline 42 & 7.99 & 352.1099 & $160.0434,174.0585,128.0526,263.1236,309.1235$ & 1.55 & 0.82 & $\uparrow$ \\
\hline 43 & 7.99 & 159.0351 & $114.0372,102.0132$ & 1.53 & 0.79 & $\uparrow$ \\
\hline 44 & 8.28 & 439.1418 & $160.0408,217.0632,100.0753,176.0653$ & 1.26 & 0.23 & $\uparrow$ \\
\hline 45 & 10.26 & 685.3406 & $120.0794,226.1537,412.1598,664.8122$ & 1.14 & 0.33 & $\uparrow$ \\
\hline 46 & 10.48 & 366.1261 & - & 1.85 & 0.34 & $\downarrow$ \\
\hline 47 & 10.56 & 815.0357 & $776.6065,173.1272,758.7957,602.3212$ & 1.24 & 0.91 & $\uparrow$ \\
\hline 48 & 14.89 & 442.2257 & - & 1.19 & 0.69 & $\uparrow \uparrow$ \\
\hline \multicolumn{7}{|c|}{ Extracellular fluid (HILIC) } \\
\hline 49 & 0.79 & 394.2886 & $277.1832,325.1807,135.0712,351.3373,247.1749$ & 1.11 & 0.77 & $\uparrow$ \\
\hline 50 & 0.8 & 376.2863 & $305.1168,227.0871,162.0785,180.0871,287.0871$ & 1.15 & 0.59 & $\uparrow \uparrow$ \\
\hline 51 & 0.81 & 366.2565 & $272.0810,283.2609,299.2612$ & 1.15 & 0.70 & $\uparrow$ \\
\hline 52 & 0.97 & 626.3375 & $172.9893,285.1105,356.9981$ & 1.15 & 0.99 & $\uparrow$ \\
\hline 53 & 2.53 & 412.1894 & - & 1.23 & 0.50 & $\uparrow$ \\
\hline 54 & 2.53 & 75.0325 & - & 1.32 & 0.98 & $\uparrow$ \\
\hline 55 & 4.08 & 308.1209 & $204.9890,266.9862,229.1300$ & 1.20 & 0.95 & $\uparrow$ \\
\hline 56 & 4.98 & 422.0732 & $278.9941,316.9850,112.9892$ & 1.33 & 1.04 & $\uparrow$ \\
\hline 57 & 7.39 & 354.0586 & $195.0517,273.0965,289.0917$ & 1.22 & 0.76 & $\uparrow$ \\
\hline 58 & 7.86 & 296.0420 & $254.9960,230.832,128.0412,274.9964$ & 1.14 & 0.18 & $\uparrow$ \\
\hline 59 & 7.87 & 425.1663 & - & 1.63 & 1.08 & $\uparrow$ \\
\hline 60 & 7.88 & 280.0682 & $128.0407,146.9652$ & 1.41 & 0.65 & $\uparrow$ \\
\hline 61 & 7.89 & 944.6605 & - & 1.15 & 0.60 & $\uparrow$ \\
\hline 62 & 7.90 & 453.1801 & - & 1.21 & 0.00 & $\uparrow$ \\
\hline 63 & 8.15 & 161.8513 & - & 1.37 & 0.94 & $\uparrow$ \\
\hline
\end{tabular}




\begin{tabular}{|c|c|c|c|c|c|c|}
\hline 64 & 11.28 & 445.1746 & - & 1.17 & 0.95 & $\uparrow$ \\
\hline 65 & 11.42 & 793.5629 & - & 1.13 & 1.05 & $\uparrow$ \\
\hline 66 & 11.58 & 297.1436 & - & 1.24 & 0.20 & $\uparrow$ \\
\hline \multicolumn{7}{|c|}{ Extracellular fluid (RPLC) } \\
\hline 67 & 0.63 & 196.0924 & $100.0808,110.0639,134.6824$ & 1.40 & 0.28 & $\uparrow$ \\
\hline 68 & 0.79 & 236.1207 & $122.0712,110.0694,164.1018,207.1207$ & 1.41 & 0.98 & $\uparrow$ \\
\hline 69 & 2.28 & 599.2759 & $583.2780,264.1288,395.8990,463.0221$ & 1.10 & 0.90 & $\uparrow$ \\
\hline 70 & 2.39 & 321.1621 & $286.1252,304.1353,322.1495,130.0854$ & 1.18 & 0.98 & $\uparrow$ \\
\hline 71 & 2.87 & 260.1362 & $142.0492,130.0507,114.0564$ & 1.10 & 1.08 & $\uparrow$ \\
\hline 72 & 3.18 & 321.1684 & $130.0844,147.1132,176.0699,2014.1375,120.0804$ & 1.11 & 1.02 & $\uparrow$ \\
\hline 73 & 3.27 & 349.1739 & $158.0898,175.1138,112.0891$ & 1.15 & 0.94 & $\uparrow$ \\
\hline 74 & 3.86 & 294.1215 & $120.0808,166.0863,186.0915$ & 1.12 & 1.09 & $\uparrow$ \\
\hline 75 & 4.14 & 453.1037 & $203.0808,185.0705,151.9828$ & 1.53 & 1.00 & $\uparrow$ \\
\hline 76 & 4.35 & 135.0688 & $118.9208,118.0701,107.0121,109.0437,120.0736$ & 1.17 & 0.99 & $\uparrow$ \\
\hline 77 & 4.56 & 321.1329 & $130.0510,176.0715,147.0775,277.1010$ & 1.16 & 1.06 & $\uparrow$ \\
\hline 78 & 4.56 & 173.0470 & $172.0377,144.0464,114.9999,130.0849,133.9231,139.0080,152.9794$ & 1.16 & 0.75 & $\uparrow$ \\
\hline 79 & 5.16 & 90.0468 & - & 1.25 & 0.94 & $\uparrow$ \\
\hline 80 & 5.16 & 272.0502 & $188.0368,232.0274,161.0255$ & 1.22 & 0.81 & $\uparrow$ \\
\hline 81 & 5.16 & 288.0222 & $149.0039,232.0287,122.0714,188.0454$ & 1.24 & 0.92 & $\uparrow$ \\
\hline 82 & 5.20 & 333.1323 & $188.0696,146.0589,215.1262,130.0497$ & 1.12 & 1.03 & $\uparrow$ \\
\hline 83 & 5.81 & 443.1182 & $269.0636,176.0749,147.0607,402.6194$ & 1.29 & 1.06 & $\uparrow$ \\
\hline 84 & 6.07 & 334.0991 & $289.1010,128.0521,159.0908,176.0703,243.0981$ & 1.75 & 0.90 & $\uparrow$ \\
\hline 85 & 6.60 & 332.0847 & $215.1296,315.0787,155.0786,199.0739$ & 1.35 & 1.06 & $\uparrow$ \\
\hline 86 & 6.90 & 276.0600 & $132.0096,213.0706$ & 1.26 & 1.04 & $\uparrow$ \\
\hline 87 & 7.00 & 542.1511 & $512.1337,301.1184,257.1279,160.0441$ & 1.15 & 0.74 & $\uparrow$ \\
\hline 88 & 7.02 & 159.0354 & $114.0375,142.0295$ & 1.16 & 0.99 & $\uparrow$ \\
\hline 89 & 7.02 & 415.0872 & $241.0298,151.9833,122.0294,176.0684$ & 1.18 & 1.00 & $\uparrow$ \\
\hline 90 & 7.02 & 240.0238 & $114.0369,160.0427,130.0865$ & 1.17 & 0.99 & $\uparrow$ \\
\hline 91 & 7.02 & 628.0435 & - & 1.12 & 1.06 & $\uparrow$ \\
\hline 92 & 7.47 & 159.0352 & 114.0378 & 1.11 & 0.99 & $\uparrow$ \\
\hline 93 & 7.47 & 574.1247 & $241.0319,416.0960,160.0431,217.0645,335.1054$ & 1.10 & 0.96 & $\uparrow$ \\
\hline 94 & 8.24 & 159.0352 & $114.0371,142.0318$ & 1.45 & 0.94 & $\uparrow$ \\
\hline 95 & 8.70 & 292.1431 & $211.0625,143.0627$ & 1.12 & 0.78 & $\uparrow$ \\
\hline 96 & 11.26 & 527.1726 & $227.5030,131.0783,201.1099,377.0110$ & 1.46 & 1.08 & $\uparrow$ \\
\hline 97 & 12.21 & 236.1207 & $160.0413,309.1310,174.0588,128.0550,219.0516$ & 1.22 & 1.05 & $\uparrow$ \\
\hline
\end{tabular}




\begin{tabular}{|c|c|c|c|c|c|c|}
\hline 98 & 12.50 & 358.1421 & $223.0476,256.2228,108.0555,350.2162$ & 1.84 & 1.01 & $\uparrow$ \\
\hline 99 & 14.88 & 229.2404 & $112.8952,212.2344,148.0868,172.9336$ & 1.13 & 0.60 & $\uparrow$ \\
\hline 100 & 17.02 & 487.2589 & $119.0836,203.9898,145.1113,365.1391$ & 1.24 & 0.74 & $\uparrow$ \\
\hline 101 & 17.07 & 257.2714 & - & 1.10 & 0.97 & $\uparrow \uparrow$ \\
\hline 102 & 19.08 & 278.1521 & 149.0228 & 1.16 & 1.06 & $\uparrow$ \\
\hline 103 & 27.78 & 227.1898 & - & 1.38 & 1.02 & $\uparrow$ \\
\hline 104 & 27.94 & 394.2134 & - & 1.10 & 0.97 & $\frac{1}{\uparrow}$ \\
\hline
\end{tabular}

* $\uparrow:$ The metabolite (on average) is more abundant in HG vs NG; $\downarrow$ : The metabolite (on average) is less abundant in HG vs NG 